\title{
On the Choice of Algebra for Quantization
}

\author{
Benjamin H. Feintzeig* \\ Department of Philosophy \\ University of Washington
}

\begin{abstract}
In this paper, I examine the relationship between physical quantities and physical states in quantum theories. I argue against the claim made by Arageorgis (1995) that the approach to interpreting quantum theories known as Algebraic Imperialism allows for "too many states". I prove a result establishing that the Algebraic Imperialist has very general resources that she can employ to change her abstract algebra of quantities in order to rule out unphysical states.
\end{abstract}

\section{Introduction}

To construct a quantum theory, one performs a procedure known as "quantization". Quantization can be thought of as having two steps: first, one constructs an abstract $\mathrm{C}^{*}$-algebra to represent the physical quantities, or observables, of the system, which must obey the canonical commutation relations, and second, one finds a representation of that algebra in the bounded operators on some Hilbert space. Much philosophical attention has been directed at this second step, as it is now well known that in many cases of physical interest, including

${ }^{*}$ I would like to thank Hans Halvorson and Jim Weatherall for helpful comments and discussions. 
quantum field theories and quantum statistical theories in the thermodynamic limit, there is not a unique Hilbert space representation of the algebra. This leads to two general interpretive options. The Hilbert Space Conservative claims that we must pick one particular Hilbert space representation of our algebra (out of the many competing ones), and use this one as our quantum theory. On the other hand, the Algebraic Imperialist asserts that we do not need any Hilbert space representations to interpret our theory, and instead can think of our physical theory as being comprised by the abstract algebra itself.

The purpose of this paper is to argue that the methods of the Algebraic Imperialist have a particular virtue, which I call adaptability. By this, I mean that one can make small, but systematic changes in the abstract algebraic framework to deal with problems that arise.

The particular problem that I am concerned with confronting from the algebraic perspective here is how to construct a quantum theory that allows for the correct space of physically possible states. In many physical theories, one can find models that appear pathological or unphysical. And indeed, it has been claimed that one of the downfalls of the algebraic framework for quantum theories is that it allows for "too many states". And so, the argument goes, one is forced to use Hilbert space methods to restrict the physical state space under consideration.

I will show in this paper, however, that one need not appeal to Hilbert space methods to perform this reduction of the state space. Instead, one can reduce the physical state space using algebraic methods alone. I will present a result that establishes precise conditions under which this reduction of the state space can be performed. This result provides a general strategy through which to use algebraic methods to construct quantum theories.

In slightly more detail, the Algebraic Imperialist advocates using the collection of states on the abstract algebra of the canonical commutation relations as the state space of our quantum theory. But there are states on this algebra that some researchers describe as unphysical. These unphysical states are often ones that cannot be represented by any density operator in the relevant Hilbert space; this means they are ruled out by the Hilbert Space Conservative, who countenances as possible only states that appear in her favored Hilbert space representation. So it seems that the Algebraic Imperialist allows for extra states that Hilbert space methods can excise from the theory.

The response I want to suggest here, on behalf of the Algebraic Imperialist, is that we have been considering the wrong algebra, at least 
for the purposes of assessing the space of physical states. There is often a different algebra that one can use, which has exactly the physical state space we were looking for. Moreover, I will show that there is a completely general and systematic way of changing the abstract algebra - the one we use to implement the canonical commutation relations - in order to reduce the physical state space. Importantly, this general procedure is appropriate for the Algebraic Imperialist because it does not use Hilbert space methods. This allows the Algebraic Imperialist a new freedom in constructing quantum theories, and it leads to a new perspective on the issues surrounding quantization. In this sense, the argument of this paper can be thought of as an argument in favor of Algebraic Imperialism because it shows how much more one can do with algebraic methods than previously thought.

But the arguments that I present need not come attached to the interpretive position of Imperialism; the results of this paper are important for anyone who wants to use a quantization procedure to construct quantum theories, including the Hilbert Space Conservative. Before one can even begin asking about the necessity of Hilbert space representations, one must grapple with the question of how to construct an appropriate $\mathrm{C}^{*}$-algebra in the first step of the quantization procedure. According to the Imperialist, this is where all of the interesting physical development happens in quantization, but even the Conservative needs to have some algebra in mind before she can take its representations. Different options for the abstract algebra of observables appear in the physics literature, but so far only one of these options has been discussed in the philosophical literature to my knowledge. As such there has been no systematic discussion of how to choose between different algebras. One contribution of this paper, independent of the dispute between Imperialists and Conservatives, will be to provide tools for choosing the appropriate algebra.

The paper is organized as follows. Section 2 briefly presents mathematical preliminaries concerning both abstract algebraic methods and Hilbert space representations. Section 3 presents the objection that Imperialism allows for "too many" states, which we take up in Section 4 . There, we present a general result concerning the reduction of the state space of an abstract algebra, and show that it specifically allows the Algebraic Imperialist at least all of the same resources that the Hilbert Space Conservative has for state space reduction. Section 5 concludes with a discussion of the significance of the results and further open questions. 


\section{Mathematical Preliminaries}

The bounded observables of a physical theory carry the structure of a $C^{*}$-algebra. ${ }^{1}$ This means that one may add and multiply observables, and multiply observables by scalars. In addition, a $\mathrm{C}^{*}$-algebra carries an operation of involution that is a generalization of complex conjugation. A $\mathrm{C}^{*}$-algebra $\mathfrak{A}$ comes equipped with a norm, which is required to satisfy the $\mathrm{C}^{*}$-identity:

$$
\left\|A^{*} A\right\|=\|A\|^{2}
$$

for all $A \in \mathfrak{A}$. The norm defines a topology, called the norm topology, which is characterized by the following condition for convergence. A net $\left\{A_{i}\right\} \subseteq \mathfrak{A}$ converges to $A$ in the norm topology ${ }^{2}$ iff

$$
\left\|A_{i}-A\right\| \rightarrow 0
$$

where the convergence is now in the standard topology on $\mathbb{R}$. The $\mathrm{C}^{*}$-algebra $\mathfrak{A}$ is required to be complete with respect to this topology in the sense that for every Cauchy net $\left\{A_{i}\right\} \subseteq \mathfrak{A}$, i.e. for every net such that

$$
\left\|A_{i}-A_{j}\right\| \rightarrow 0
$$

there is an $A \in \mathfrak{A}$ such that $A_{i} \rightarrow A$ in the norm topology. Standard results in the theory of normed vector spaces tell us that every normed vector space has a unique completion. ${ }^{3}$

Since $\mathfrak{A}$ is a vector space, we can also consider the dual space $\mathfrak{A}^{*}$ of bounded (i.e. norm continuous) linear functionals $\rho: \mathfrak{A} \rightarrow \mathbb{C}$. A state on a $\mathrm{C}^{*}$-algebra $\mathfrak{A}$ is just a particular kind of element of the dual space $\mathfrak{A}^{*}$ —namely one that is positive and normalized. ${ }^{4}$

\footnotetext{
${ }^{1}$ In this section, we present only the minimal technical background required to state the results of Section $\S 4$. For more on operator algebras, see Kadison and Ringrose (1997), Sakai (1971), and Landsman (1998). For more on algebraic quantum theory, see Haag (1992), Bratteli and Robinson (1996), Emch (1972), and Wald (1994). For philosophical introductions, see Halvorson (2006) and Ruetsche (2011).

${ }^{2}$ One could restrict attention here to sequences because the norm topology is second countable, but for the weak topologies considered later, which are not second countable, one must work with arbitrary nets.

${ }^{3} \mathrm{~A}$ complete normed vector space is called a Banach space. A C $\mathrm{C}^{*}$-algebra is thus a Banach algebra whose norm is, in a certain sense, compatible with multiplication and involution.

${ }^{4} \mathrm{~A}$ linear functional $\rho \in \mathfrak{A}^{*}$ is positive if $\rho\left(A^{*} A\right) \geq 0$ for all $A \in \mathfrak{A}$ and normalized if $\|\rho\|=1$.
} 
The dual space $\mathfrak{A}^{*}$ can be used to define an alternative to the norm topology on $\mathfrak{A}$, called the weak topology, which is characterized by the following condition for convergence. A net $\left\{A_{i}\right\} \subseteq \mathfrak{A}$ converges in the weak topology to $A \in \mathfrak{A}$ iff for every $\rho \in \mathfrak{A}^{*}$,

$$
\rho\left(A_{i}\right) \rightarrow \rho(A)
$$

where the convergence is now in the standard topology on $\mathbb{C}$. The weak topology is the coarsest topology on $\mathfrak{A}$ with respect to which all of the linear functionals in $\mathfrak{A}^{*}$ are continuous.

A $\mathrm{C}^{*}$-algebra $\mathfrak{A}$ need not be complete with respect to its weak topology; there may be nets $\left\{A_{i}\right\} \subseteq \mathfrak{A}$ that are Cauchy in the sense that

$$
\rho\left(A_{i}-A_{j}\right) \rightarrow 0
$$

for every $\rho \in \mathfrak{A}^{*}$ without the net having a limit point $A \in \mathfrak{A}$ such that $A_{i} \rightarrow A$ in the weak topology. However, a $\mathrm{C}^{*}$-algebra can always be completed in its weak topology to form its bidual $\mathfrak{A}^{* *}$, as follows. ${ }^{5}$

The bidual carries a topology known as the weak* topology, which is a natural generalization of the weak topology on $\mathfrak{A}$. The weak* topology is characterized by the following condition for convergence. A net $\left\{A_{i}\right\} \subseteq \mathfrak{A}^{* *}$ converges in the weak* topology to $A \in \mathfrak{A}^{* *}$ iff for every $\rho \in \mathfrak{A}^{*}$,

$$
A_{i}(\rho) \rightarrow A(\rho)
$$

The weak* topology on the bidual $\mathfrak{A}^{* *}$ corresponds precisely to the extension of the condition of convergence for the weak topology on $\mathfrak{A}$ to the larger algebra $\mathfrak{A}^{* *}$. In particular, the weak* topology on $\mathfrak{A}^{* *}$ is the coarsest topology on $\mathfrak{A}^{* *}$ that makes every linear functional in $\mathfrak{A}^{*}$ continuous. One can show that the bidual $\mathfrak{A}^{* *}$ is complete with respect to the weak* topology.

Moreover, the original $\mathrm{C}^{*}$-algebra $\mathfrak{A}$ is canonically embedded in its bidual by $A \in \mathfrak{A} \mapsto \hat{A} \in \mathfrak{A}^{* *}$, with $\hat{A}$ defined by

$$
\hat{A}(\rho)=\rho(A)
$$

for all $\rho \in \mathfrak{A}^{*}$. With respect to this embedding, $\mathfrak{A}$ is dense in $\mathfrak{A}^{* *}$ in the weak* topology, so the bidual $\mathfrak{A}^{* *}$ can be understood as the completion of $\mathfrak{A}$ in its weak topology, which is the subspace topology of the weak* topology on $\mathfrak{A}^{* *}$.

\footnotetext{
${ }^{5}$ See Feintzeig $(2016 \mathrm{~b})$ for more on the completion of a $\mathrm{C}^{*}$-algebra into its bidual.
} 
Importantly, the algebraic approach can be translated back into the familiar Hilbert space formalism for quantum mechanics. A representation of a $\mathrm{C}^{*}$-algebra $\mathfrak{A}$ is a pair $(\pi, \mathcal{H})$, where $\mathcal{H}$ is a Hilbert space and $\pi: \mathfrak{A} \rightarrow \mathcal{B}(\mathcal{H})$ is a ${ }^{*}$-homomorphism into the bounded linear operators on $\mathcal{H}^{6}$

We can use the Hilbert space structure of a representation $(\pi, \mathcal{H})$ to induce a new topology on the algebra $\pi(\mathfrak{A})$. The ultraweak topology is characterized through the following condition for convergence: a net $\pi\left(A_{i}\right)$ converges to $\pi(A)$ in the ultraweak topology if for every density operator $\rho$ on $\mathcal{H}$,

$$
\operatorname{Tr}\left(\pi\left(A_{i}\right) \rho\right) \rightarrow \operatorname{Tr}(\pi(A) \rho)
$$

The ultraweak topology represents a notion of convergence of expectation values by certain states.

A state $\omega \in \mathfrak{A}^{*}$ has a density operator representative in the representation $(\pi, \mathcal{H})$ just in case there is a density operator $\rho_{\omega}$ such that $\omega(A)=\operatorname{Tr}\left(A \rho_{\omega}\right)$ for all $A \in \mathfrak{A}$. In general, there may be states on $\mathfrak{A}$ without density operator representatives in a given representation $(\pi, \mathcal{H})$. In other words, the density operator states on a representation may not exhaust the states on the abstract algebra $\mathfrak{A} .{ }^{7}$ However, we know that a state $\omega \in \mathfrak{A}^{*}$ has a density operator representative in a given representation $(\pi, \mathcal{H})$ of $\mathfrak{A}$ just in case $\omega$ is ultraweakly continuous in that representation. We will use these facts in our discussion of appropriate state spaces below.

\section{$3 \quad$ Interpretive Options}

The procedure of quantization can be understood as involving two steps. First, one finds a quantum algebra of observables $\mathfrak{A}$. Second, one chooses a representation $(\pi, \mathcal{H})$ of $\mathfrak{A}$ on some Hilbert space. The interpretive debate between Algebraic Imperialism and Hilbert Space Conservatism concerns just whether this second step is necessary. ${ }^{8}$ According to the Algebraic Imperialist, it is not: a quantum theory

\footnotetext{
${ }^{6}$ One of the most fundamental results in the theory of $\mathrm{C}^{*}$-algebras, known as the GNS Theorem (See Kadison and Ringrose, 1997), tells us that every C*-algebra has Hilbert space representations.

${ }^{7}$ This follows immediately from the prensence of unitarily inequivalent representations of an algebra, as discussed in Ruetsche (2011).

${ }^{8}$ For more on Algebraic Imperialism and Hilbert Space Conservatism, see Ruetsche (2002, 2003, 2006, 2011).
} 
(or, at least its kinematics) can be captured by the abstract algebra $\mathfrak{A}$ in the sense that the physical quantities of a system can be adequately represented by the elements of $\mathfrak{A}$ - or perhaps by weak limits in $\mathfrak{A}^{* *}$ and the physical states can be represented by states on $\mathfrak{A}$. On the other hand, according to the Hilbert Space Conservative, we need to pick a representation $(\pi, \mathcal{H})$. Then we represent the physical quantities of our system by elements of $\pi(\mathfrak{A})$ - or perhaps by ultraweak limits - and the physical states by density operators on $\mathcal{H}$.

The objection that has been leveled at Algebraic Imperialism that I want to discuss is that the Imperialist allows for "too many" states, in the sense that many states on the abstract algebra $\mathfrak{A}$ are unphysical (See Arageorgis, 1995). Taking a Hilbert space representation allows us to focus on an appropriate collection of physical states by focusing our attention on only the states that have density operator representatives in our chosen representation. So the fact that Hilbert space methods provide resources to rule out unphysical states is supposed to count in favor of the Hilbert Space Conservative.

Before we can see what these unphysical states are, we need to specify the algebra of observables we are using - after all, different algebras will in general have different state spaces. The algebra of observables that gives rise to the unphysical states at issue is known as the Weyl Algebra. This algebra puts the canonical commutation relations between position and momentum observables in bounded form by considering only exponentiated forms of those observables. ${ }^{9}$

The states that one might consider unphysical come in may varieties. For example, the Weyl algebra allows for non-regular states (Halvorson, 2001, 2004; Beaume et al., 1974), ones which fail to satisfy a continuity condition and in doing so fail to allow one to simultaneously define both position and momentum observables from the Weyl operators. These non-regular states do not have density operator representatives in the usual Hilbert space representation (the Schrödinger representation) of the Weyl algebra. It is a standard move in algebraic quantum theory to restrict attention only to regular states on the Weyl algebra and their representations to rule out these non-regular states.

But there are many other states on the Weyl algebra that one might consider ruling out as unphysical. Arageorgis (1995) mentions a pro-

\footnotetext{
${ }^{9}$ These Weyl operators take the form $U(a):=e^{i a Q}$ and $V(b):=e^{i b P}$ for position $Q$, momentum $P$, and constants $a, b \in \mathbb{R}$ (at least in the usual Schrödinger representation). For more on the Weyl algebra, see Petz (1990) and Clifton and Halvorson (2001). I save more detailed application of results in Section $\S 4$ to the Weyl algebra for future work.
} 
posal that we ought to restrict attention to locally definite states, ones which vanish on smaller and smaller regions of spacetime. Or perhaps we ought to restrict attention to Hadamard states, ones which allow for an appropriately well-defined stress-energy observable (See Wald, 1994). And Halvorson (2006) takes up a suggestion by Doplicher, Haag, and Roberts that we use only what he calls DHR states, ones which differ only locally from the vacuum. In addition, we might want to restrict attention to Fock states, ones that allow for an interpretation in terms of particle states with creation and annihilation operators (See Petz, 1990). For the purposes of this paper, I will not analyze these suggestions in detail. The results that I present in the next section are meant to be sufficiently general to apply to any of these suggestions for appropriate physical state spaces, although further work is required to make these connections explicit.

Furthermore, I should stress that it is not my purpose here to judge whether any of the proposals cited above provide an adequate specification of the states that we ought to deem physical. I mention these concrete proposals only to show that others have expressed an interest in restricting the space of quantum states. My goal in this paper is only to show that however one wants to specify the collection of physical states, there is an intimate relationship between this state space and the algebra of observables of the quantum theory.

The fact that the abstract Weyl algebra allows for so many supposedly unphysical states has been taken by some (e.g., Arageorgis, 1995) as an argument against Algebraic Imperialism. However, I propose that this only gives us reason to use a different algebra. Even if one particular algebra allows for unphysical states, this does not imply that all abstract algebras allow for unphysical states. There are other options for the abstract algebra of observables.

In the next section, I will present general algebraic results that allow us to change the algebra implementing the canonical commutation relations in order to restrict its physical state space. But before I present these results, I want to note that we already have a procedure, at least in the case of a simple system with finitely many degrees of freedom, ${ }^{10}$ for eliminating unphysical states by choosing an appropriate algebra. For these simple systems, it is standard to restrict attention to only regular states because a result known as the Stone-von Neumann Theorem tells us that there is a unique irreducible represen-

\footnotetext{
${ }^{10}$ Specifically, the procedure will work for quantizing a classical system with finitely many degrees of freedom and simply connected phase space $\mathbb{R}^{2 n}$.
} 
tation $^{11}$ of the Weyl algebra in which all of the regular states (and, it turns out, only the regular states) have density operator representatives. This representation is just the usual Schrödinger representation, and in this representation the ultraweak closure of the Weyl algebra is the algebra $\mathcal{B}(\mathcal{H})$. So this leads us back to the familiar setting for nonrelativistic quantum mechanics, where every (self-adjoint) element of $\mathcal{B}(\mathcal{H})$ is considered as a physically significant observable and every physical state has a density operator representative. ${ }^{12}$

However, for these simple systems with finitely many degrees of freedom, there is another path one can take to get the same theory without using Hilbert space methods. ${ }^{13}$ Instead of choosing the Weyl algebra, one can directly use the algebra of compact operators on a separable Hilbert space (i.e., $\left.L^{2}(\mathbb{R})\right) .{ }^{14}$ Then one immediately finds that the state space of this algebra is equivalent to the collection of regular states. One way to see this is to notice that the compact operators have a unique irreducible representation, ${ }^{15}$ so one has an immediate analog of the Stone-von Neumann Theorem. The bidual of the algebra of compact operators is its ultraweak closure in this representation, the familiar algebra $\mathcal{B}(\mathcal{H})$. And there are no non-regular or otherwise unphysical states on the algebra of compact operators that cannot be represented as density operators on this representation.

Thus, one can construct the same quantum theory that we get by using the Weyl algebra and the Stone von-Neumann theorem by instead using the compact operators and forgoing the need to restrict attention to some subspace of states. This procedure provides a route for the Algebraic Imperialist to arrive at the same collection of physical states the Hilbert Space Conservative arrives at. The key to this procedure is an auspicious choice of algebra of observables for our quantum theory. So, I suggest that one might pay closer attention to the choice of algebra in quantization procedures in other cases when

\footnotetext{
${ }^{11} \mathrm{~A}$ representation $(\pi, \mathcal{H})$ of a $\mathrm{C}^{*}$-algebra $\mathfrak{A}$ is irreducible if the only subspaces of $\mathcal{H}$ that $\pi(\mathfrak{A})$ leaves invariant are $\{0\}$ and $\mathcal{H}$.

${ }^{12}$ See Summers (1999) or Petz (1990) for more on the Stone-von Neumann theorem and the Schrödinger representation of the Weyl algebra.

${ }^{13}$ For more on this algebraic approach to regular states, see Feintzeig (2016a).

${ }^{14}$ For example, one might arrive at this algebra through the prescription known as Berezin quantization (Landsman, 1998, 2006). If one is worried that one needs a Hilbert space to define this algebra, note that Landsman (1990) provides a way to understand this construction from a purely $\mathrm{C}^{*}$-algebraic point of view.

${ }^{15}$ See Thm. 10.4.6 of Kadison and Ringrose (1997, p. 751).
} 
constructing quantum theories. The next section shows that if one takes seriously the importance of this choice of algebra, then one can develop powerful algebraic tools generalizing this procedure for representing physical systems with specified state spaces.

\section{Algebraic Adaptability}

The purpose of this section is to develop a general response on behalf of the Algebraic Imperialist to the objection that the abstract algebra allows for "too many states". First, in $\S 4.1$ we prove a general result providing necessary and sufficient conditions under which one can find a $\mathrm{C}^{*}$-algebra with a restricted state space. This allows the Algebraic Imperialist far more flexibility than previously thought in constructing a theory with the appropriate state space. Next, in $\S 4.2$ we illustrate how this result can be applied to transform any algebra sufficiently similar to the Weyl algebra to the algebra $\mathcal{B}(\mathcal{H})$ of all bounded operators on a separable Hilbert space. This shows that the Algebraic Imperialist has at least as much power as the Hilbert Space Conservative to limit the collection of states she deems physical. The Algebraic Imperialist always has the option to choose the collection of states with density operator representatives in a given Hilbert space representation as her priveleged collection of states and then apply the results here to find a new algebra with precisely that state space.

\subsection{General Algebraic Results}

Suppose that we have a $\mathrm{C}^{*}$-algebra $\mathfrak{A}$ and some preferred subset of its dual space $V \subseteq \mathfrak{A}^{*}$. This section shows necessary and sufficient conditions for the existence of an algebra inheriting "the same" algebraic relations but with $V$ as its entire dual space.

First we will need some conditions to ensure that $V$ can be dual to a space supporting an appropriate $\mathrm{C}^{*}$-norm. To that end, define $\|\cdot\|_{V}: \mathfrak{A} \rightarrow \mathbb{R}$ as follows for all $A \in \mathfrak{A}:$

$$
\|A\|_{V}=\sup _{\omega \in V ;\|\omega\|=1}|\omega(A)|
$$

With this definition, consider the following conditions on $V$ :

(i) For all $A \in \mathfrak{A}$,

$$
\left\|A^{*} A\right\|_{V}=\|A\|_{V}^{2}
$$


(ii) For all $A, B \in \mathfrak{A}$,

$$
\|A B\|_{V} \leq\|A\|_{V}\|B\|_{V}
$$

(iii) If $\left\{A_{n}\right\} \subseteq \mathfrak{A}$ is a sequence such that

$$
\lim _{n, m \rightarrow \infty}\left\|A_{n}-A_{m}\right\|_{V}=0
$$

then there is an element $A \in \mathfrak{A}$ such that

$$
\lim _{n \rightarrow \infty}\left\|A_{n}-A\right\|_{V}=0
$$

The first two conditions are minimal technical conditions that ensure $V$ can be the dual space to a $\mathrm{C}^{*}$-algebra: condition (i) ensures that $V$ can be dual to an algebra satisfying the $\mathrm{C}^{*}$-identity, and (ii) ensures that $V$ can be dual to an algebra whose multiplication operation is norm continuous. Condition (iii) ensures that $V$ can be dual to an algebra that is complete in norm, but we can consider this as a technical redundancy because every normed vector space has a unique completion.

We need one more piece of background before we can present the main result. The following piece of apparatus allows us to ensure that $V$ has enough structure to support algebraic operations inherited from $\mathfrak{A}$, as we will make precise in a moment. For any $\omega \in \mathfrak{A}^{*}$, define a relation $\sim_{\omega}$ on $\mathfrak{A}$ by

$A \sim_{\omega} B$ iff for all $C \in \mathfrak{A}, \omega(A C)=\omega(B C)$ and $\omega(C A)=\omega(C B)$

And define a relation $\sim_{V}$ on $\mathfrak{A}$ by

$$
A \sim_{V} B \text { iff for all } \omega \in V, A \sim_{\omega} B
$$

One easily checks that for any $\omega \in \mathfrak{A}^{*}$, the relation $\sim_{\omega}$ is an equivalence relation, and similarly for any $V \subseteq \mathfrak{A}^{*}$, the relation $\sim_{V}$ is an equivalence relation. Now consider the following condition:

(iv) $V$ is maximal in the sense that

$$
V=\left\{\omega \in \mathfrak{A}^{*}: \text { for all } A, B \in \mathfrak{A} \text {, if } A \sim_{V} B \text {, then } A \sim_{\omega} B\right\}
$$

Condition (iv) guarantees that $V$ is as big as possible, by containing all states compatible with the states in $V$. As we will see, condition (iv) is used to ensure that $V$ is "big enough" to support the algebraic 
operations of $\mathfrak{A}$. But, like condition (iii), it can be seen as a technical redundancy because we can generate a maximal subspace from any other subspace $V_{0} \subseteq \mathfrak{A}^{*}$ by the following proposition. ${ }^{16}$

Proposition 1. Let $\mathfrak{A}$ be a $C^{*}$-algebra and let $V_{0} \subseteq \mathfrak{A}^{*}$. Let

$$
V=\left\{\omega \in \mathfrak{A}^{*}: \text { for all } A, B \in \mathfrak{A} \text {, if } A \sim_{V_{0}} B \text {, then } A \sim_{\omega} B\right\}
$$

Then $V$ is maximal in the sense of condition (iv) above, i.e.

$$
V=\left\{\omega \in \mathfrak{A}^{*}: \text { for all } A, B \in \mathfrak{A}, \text { if } A \sim_{V} B, \text { then } A \sim_{\omega} B\right\}
$$

In this case, we will say that the vector space $V$ is generated by $V_{0}$. Given a collection of states in $V_{0}, V$ is the smallest maximal collection of states containing $V_{0}$. Now we are ready to present our main result, which says that the conditions listed above are necessary and sufficient for reducing the state space of our algebra.

Theorem 1. Let $\mathfrak{A}$ be a $C^{*}$-algebra and let $V \subseteq \mathfrak{A}^{*}$. Then there exists a $C^{*}$-algebra $\mathfrak{B}$ and a surjective ${ }^{*}$-homomorphism $f: \mathfrak{A} \rightarrow \mathfrak{B}$ such that $\mathfrak{B}^{*} \cong V$ with the isomorphism given by $\omega \in \mathfrak{B}^{*} \mapsto(\omega \circ f) \in V$ iff conditions (i)-(iv) are satisfied.

The main idea of the proof (contained in the appendix) is to construct the $\mathrm{C}^{*}$-algebra $\mathfrak{B}$ by taking the quotient of the original algebra $\mathfrak{A}$ by the equivalence relation $\sim_{V}$; in other words, $\mathfrak{B}=\mathfrak{A} / \sim_{V}$. Moreover, we can show that the algebra we get through this construction is, in a certain sense, unique.

Theorem 2. Let $(\mathfrak{B}, f)$ be the pair given by Thm. 1. For any other $C^{*}$-algebra $\mathfrak{C}$ and surjective ${ }^{*}$-homomorphism $g: \mathfrak{A} \rightarrow \mathfrak{C}$ such that $\mathfrak{C}^{*} \cong V$ with the isomorphism given by $\omega \in \mathfrak{C}^{*} \mapsto(\omega \circ g) \in V$, there is $a{ }^{*}$-isomorphism $\alpha: \mathfrak{B} \rightarrow \mathfrak{C}$ such that $\alpha \circ f=g$.

These theorems show that one can specify the physical state space however one likes, as long as it satisfies conditions (i)-(iv). Whenever these conditions are satisfied (and only when these conditions are satisfied) we can find a new algebra $\mathfrak{A} / \sim_{V}$ that inherits the algebraic relations of $\mathfrak{A}$ through the surjective *-homomorphism $f$, and this new algebra has precisely the physical state space as its entire state space.

\footnotetext{
${ }^{16}$ Proofs of all results are contained in the appendix.
} 


\subsection{Example: $\mathcal{B}(\mathcal{H})$}

In this section we deal with the same simple quantum theories of systems with finitely many degrees of freedom considered in $\S 3$. Such a theory has as physical states the density operators on a Hilbert space $\mathcal{H}$ with observables given by all operators in $\mathcal{B}(\mathcal{H})$. But suppose that we've chosen some other algebra of quantum observables. Let $\mathfrak{A}$ be any $\mathrm{C}^{*}$-subalgebra of $\mathcal{B}(\mathcal{H})$ that contains the constants and separates density operator states in the sense that for any two density operators $\rho_{1}, \rho_{2}$ on $\mathcal{H}$, there is an $A \in \mathfrak{A}$ such that $\operatorname{Tr}\left(A \rho_{1}\right) \neq \operatorname{Tr}\left(A \rho_{2}\right)$. The Schrödinger representation of the Weyl algebra mentioned in the previous section is an example of just such an algebra. Here we show that there is a natural way to transform $\mathfrak{A}$ into all of $\mathcal{B}(\mathcal{H})$ using purely algebraic methods.

Why do we need such a procedure, given that we can just take the ultraweak closure of the algebra $\mathfrak{A}$ in the natural inclusion representation on $\mathcal{H}$ to get all of $\mathcal{B}(\mathcal{H}) ?^{17}$ Of course, we could obtain all of $\mathcal{B}(\mathcal{H})$ in this way, but there is something unsatisfying about this approach from the algebraic perspective. Why should we complete $\mathfrak{A}$ in the ultraweak topology when there may be states on $\mathfrak{A}$ that are not ultraweakly continuous? There's a sense in which completing $\mathfrak{A}$ in the ultraweak topology does not respect the algebraic structure (or really the state space) of $\mathfrak{A}$, because the completion of $\mathfrak{A}$ in its abstract weak topology (as opposed to the ultraweak topology) to form the bidual $\mathfrak{A}^{* *}$ in fact leads us to a much larger algebra than $\mathcal{B}(\mathcal{H}) .{ }^{18}$

I will show that there is a general procedure one can perform by applying Thm. 1 to transform $\mathfrak{A}$ into $\mathcal{B}(\mathcal{H})$. To see this, notice that there is a sense in which $\mathfrak{A}$ is too small and a sense in which it is too large. $\mathfrak{A}$ is too small in the sense that it may not contain many elements of $\mathcal{B}(\mathcal{H})$ like projections. But $\mathfrak{A}$ is too large in the sense that it may allow for states that are not ultraweakly continuous and so cannot be represented by density operators on $\mathcal{H}$. As such, we will first enlarge $\mathfrak{A}$ to $\mathfrak{A}^{* *}$ to obtain all of the missing operators including the projections on $\mathcal{H}$. Then we will restrict attention to an appropriate collection of physical states by applying Thm. 1 .

Let $V_{0}^{Q}$ be the collection of bounded linear functionals on $\mathfrak{A}$ that

\footnotetext{
${ }^{17}$ Notice that the natural inclusion representation of the algebra $\mathfrak{A}$ on $\mathcal{H}$ is irreducible and employ Prop. 1.21.9 of (Sakai, 1971, p. 52).

${ }^{18}$ More precisely, the algebra $\mathcal{B}(\mathcal{H})$ will in general be properly embedded in the $\mathfrak{A}^{* *}$, which one can see directly in the universal representation of $\mathfrak{A}$ (See Feintzeig, 2016b).
} 
are ultraweakly continuous on $\mathcal{H}$. The following proposition shows that reducing $\mathfrak{A}^{* *}$ by Thm. 1 with this preferred collection of states brings us back to the usual setting of $\mathcal{B}(\mathcal{H})$.

Proposition 2. Let $V_{Q}$ be the vector subspace of bounded linear functionals on $\mathfrak{A}^{* *}$ generated by $V_{0}^{Q}$ Then the $C^{*}$-algebra $\mathfrak{A}^{* *} / \sim_{V_{Q}}$ in Thm. 1 is *-isomorphic to $\mathcal{B}(\mathcal{H})$.

This shows that one can generate $\mathcal{B}(\mathcal{H})$ by first adding to $\mathfrak{A}$ the (weak) limit observables in $\mathfrak{A}^{* *}$ and then reducing the algebra by Thm. 1 to restrict attention to only states with density operator representatives on $\mathcal{H}$. Again, the first step of enlarging the algebra to $\mathfrak{A}^{* *}$ is necessary because there may be many bounded operators outside $\mathfrak{A}$. In particular, we cannot reduce $\mathfrak{A}$ directly to the compact operators (recall that $\mathcal{B}(\mathcal{H})$ is the bidual and hence ultraweak closure of the compact operators) because there may be compact operators that are not in $\mathfrak{A}$, as in the case of representations of the Weyl algebra.

This shows that the Algebraic Imperialist always has the resources to reduce the physical state space of her algebra in at least all of the ways the Hilbert Space Conservative can. Where the Hilbert Space Conservative would take an irreducible representation of the algebra and restrict attention to density operator states in that representation, the Algebraic Imperialist can just choose that vector space of states (i.e., the density operator states in that representation) as her priveleged collection of states and directly apply Thm. 1 to obtain a new algebra with precisely this state space.

\section{Discussion}

I have argued that Thm. 1 gives a general tool for the Algebraic Imperialist to use to respond to the "too many states" objection. I have shown that there is a very general procedure the Algebraic Imperialist can use to rid herself of unphysical states. The result in Prop. 2 shows that this procedure can apply to any collection of physical states that consists of all and only the density operator states on some Hilbert space representation. Specifically, this procedure applies to the regular states on the Weyl algebra, which exhaust the density operator states in the Schrödinger representation. But it is still an open question whether the Algebraic Imperialist can apply this procedure to other existing candidates for physical state spaces. In other words, it is still 
left to be shown that standard proposals for physical state spaces (e.g., the Hadamard states, locally definite states, or Fock states) actually satisfy conditions (i)-(iv) of Thm. 1. Answering this question would inform us about precisely how much freedom the Imperialist has in responding to the objection that she allows for "too many states".

The results of this paper show that the Algebraic Imperialist has at least as much flexibility for restricting the state space as does the Hilbert Space Conservative. Prop. 2 shows that when the physical states themselves form the space of density operator states on a Hilbert space representation, then it is possible to reduce the algebra to one with an appropriate state space. But I have also claimed that the flexibility or adaptability the Algebraic Imperialist gains through Thm. 1 is a virtue of the algebraic point of view. As such, one ought to ask whether Thm. 1 gives us more freedom for reducing the state space than the Hilbert Space Conservative has. In other words, are there any subspaces of states $V$ satisfying (i)-(iv) that do not form the space of density operator states of some Hilbert space representation? If not, then the procedure I have outlined for reducing the state space of an abstract algebra works in exactly the same cases that the Conservative's procedure would work. Thus, I have not yet made the case that this virtue I have brought to our attention-adaptability of the algebra and state space - is a virtue of Imperialism over Conservatism. My results do show that the Imperialist can deal with the objection that she allows for "too many" states, but I admit that this may just bring the Imperialist in line with the Hilbert Space Conservative.

Even with these open questions, I believe the results of this paper have significance for the interpretation of algebraic quantum theories. They show that considerations of the physical content of the abstract algebra, its states, and topologies, can lead us to new technical and conceptual tools beyond those of Hilbert space representations. I can only hope these tools will prove useful for understanding algebraic quantum theories.

\section{Appendix: Proofs of results}

In this appendix, we prove the results of $\S 4$. The arguments rely on some technical notions not defined in the body of the paper; for explicit definitions, see Kadison and Ringrose (1997). First, we prove the results of $\S 4.1$. 
Proposition 1. Let $\mathfrak{A}$ be a $C^{*}$-algebra and let $V_{0} \subseteq \mathfrak{A}^{*}$. Define $V$ by

$$
V=\left\{\omega \in \mathfrak{A}^{*}: \text { for all } A, B \in \mathfrak{A} \text {, if } A \sim_{V_{0}} B \text {, then } A \sim_{\omega} B\right\}
$$

Then $V$ is maximal in the sense of condition (iv) above, i.e.

$$
V=\left\{\omega \in \mathfrak{A}^{*}: \text { for all } A, B \in \mathfrak{A}, \text { if } A \sim_{V} B \text {, then } A \sim_{\omega} B\right\}
$$

Proof. ( $\subseteq$ ) Suppose $\omega \in V$. If $A, B \in \mathfrak{A}$ are such that $A \sim_{V} B$, then $A \sim \sim_{V_{0}} B$ since $V_{0} \subseteq V$. It follows from the definition of $V$ that $A \sim{ }_{\omega} B$. Hence,

$$
\omega \in\left\{\omega \in \mathfrak{A}^{*}: \text { for all } A, B \in \mathfrak{A} \text {, if } A \sim_{V} B \text {, then } A \sim_{\omega} B\right\}
$$

() Suppose $\omega \in\left\{\omega \in \mathfrak{A}^{*}\right.$ : for all $A, B \in \mathfrak{A}$, if $A \sim_{V} B$, then $\left.A \sim_{\omega} B\right\}$. Suppose $A, B \in \mathfrak{A}$ are such that $A \sim_{V_{0}} B$. Let $\rho \in V$. Then, by the definition of $V, A \sim_{\rho} B$. Since this holds for all $\rho \in V$, it follows that $A \sim_{V} B$. This implies, by the assumption on $\omega, A \sim_{\omega} B$. And by the definition of $V$, it follows that $\omega \in V$.

Theorem 2. Let $\mathfrak{A}$ be a $C^{*}$-algebra and let $V \subseteq \mathfrak{A}^{*}$. Then there exists a $C^{*}$-algebra $\mathfrak{B}$ and a surjective ${ }^{*}$-homomorphism $f: \mathfrak{A} \rightarrow \mathfrak{B}$ such that $\mathfrak{B}^{*} \cong V$ with the isomorphism given by $\omega \in \mathfrak{B}^{*} \mapsto(\omega \circ f) \in V$ iff conditions (i)-(iv) are satisfied.

Proof. First, we show that the conditions (i)-(iv) are jointly sufficient. Suppose that $V \subseteq \mathfrak{A}^{*}$ satisfies conditions (i)-(iv). One easily checks that condition (iv) implies that $V$ is a norm closed subspace of $\mathfrak{A}^{*}$.

Let $\mathfrak{B}=\mathfrak{A} / \sim_{V}$, whose elements will be denoted $[A] \in \mathfrak{B}$ for each $A \in \mathfrak{A}$. We define algebraic operations on $\mathfrak{B}$ as follows:

$$
\begin{aligned}
{[A]+[B] } & :=[A+B] \\
\alpha[A] & :=[\alpha A] \\
{[A]^{*} } & :=\left[A^{*}\right] \\
{[A][B] } & :=[A B]
\end{aligned}
$$

(The definition of $\sim_{V}$ guarantees that multiplication is well-defined.)

Let $f: \mathfrak{A} \rightarrow \mathfrak{B}$ be defined by: for all $A \in \mathfrak{A}$,

$$
f(A):=[A]
$$

Define a norm on $\mathfrak{B}$ by $[A] \in \mathfrak{B}$,

$$
\|[A]\|=\|A\|_{V}
$$


for all $A \in \mathfrak{A}$. One easily checks that this is well-defined and indeed satisfies the conditions for being a norm.

Now for any $\omega \in \mathfrak{B}^{*}$, it follows from condition (iv) that $\omega \circ f \in$ $V$. Hence, it is easy to check that $\omega \in \mathfrak{B}^{*} \mapsto(\omega \circ f) \in V$ is an isomorphism.

Now, we show that $\mathfrak{B}$ is a $\mathrm{C}^{*}$-algebra with the above operations. Condition (ii) implies that multiplication on $\mathfrak{B}$ is norm continuous because for any $[A],[B] \in \mathfrak{B}$,

$$
\|[A B]\|=\|A B\|_{V} \leq\|A\|_{V}\|B\|_{V}=\|[A]\|\|[B]\|
$$

Condition (i) implies that the $\mathrm{C}^{*}$-identity is satisfied because for any $[A] \in \mathfrak{B}$,

$$
\left\|[A]^{*}[A]\right\|=\left\|A^{*} A\right\|_{V}=\|A\|_{V}^{2}=\|[A]\|^{2}
$$

Finally, condition (iii) implies that $\mathfrak{B}$ is complete with respect to this norm because if $\left\{\left[A_{n}\right]\right\} \subseteq \mathfrak{B}$ is a Cauchy sequence, then

$$
\lim _{n, m \rightarrow \infty}\left\|A_{n}-A_{m}\right\|_{V}=\lim _{n, m \rightarrow \infty}\left\|\left[A_{n}\right]-\left[A_{m}\right]\right\|=0
$$

and hence there is an $A \in \mathfrak{A}$ such that

$$
\lim _{n \rightarrow \infty}\left\|A_{n}-A\right\|_{V}=0
$$

from which it follows immediately that

$$
\lim _{n \rightarrow \infty}\left\|\left[A_{n}\right]-[A]\right\|=0
$$

One easily checks now that $f$ is indeed a surjective *-homomorphism.

It is also easy to check that each of the conditions (i)-(iv) is necessary in order to guarantee that $\mathfrak{B}$ is indeed a $\mathrm{C}^{*}$-algebra.

Theorem 3. Let $(\mathfrak{B}, f)$ be the pair given by Thm. 1. For any other $C^{*}$-algebra $\mathfrak{C}$ and surjective ${ }^{*}$-homomorphism $g: \mathfrak{A} \rightarrow \mathfrak{C}$ such that $\mathfrak{C}^{*} \cong V$ with the isomorphism given by $\omega \in \mathfrak{C}^{*} \mapsto(\omega \circ g) \in V$, there is $a{ }^{*}$-isomorphism $\alpha: \mathfrak{B} \rightarrow \mathfrak{C}$ such that $\alpha \circ f=g$.

Proof. Suppose $\mathfrak{C}$ is a $\mathrm{C}^{*}$-algebra with surjective ${ }^{*}$-homomorphism $g: \mathfrak{A} \rightarrow \mathfrak{C}$ such that $\mathfrak{C}^{*} \cong V$ with the isomorphism given by $\omega \in \mathfrak{C}^{*} \mapsto$ $(\omega \circ g) \in V$. Then define $\alpha: \mathfrak{B} \rightarrow \mathfrak{C}$ by $\alpha([A])=g(A)$ for all $[A] \in \mathfrak{B}$ (one requires the axiom of choice to choose a representative $A$ for each $[A] \in \mathfrak{B}$ ). One easily checks that $\alpha$ is well-defined (because $A \sim_{V} B$ implies $\|g(A)-g(B)\|=0$, which implies $g(A)=g(B))$ and a *isomorphism. Furthermore, it follows immediately that $\alpha \circ f=g$. 
Now we prove the results of $\S 4.2$. Here, $\mathfrak{A}$ is a $\mathrm{C}^{*}$-subalgebra of $\mathcal{B}(\mathcal{H})$ containing the constants and separating density operator states. Let $\pi$ denote the representation $\pi(A)=\pi_{U}(A) P$ of $\mathfrak{A}$ on $\mathcal{H}_{U}$, where $\left(\pi_{U}, \mathcal{H}_{U}\right)$ is the universal representation and $P$ is the projection determined through Thm. 10.1.12 of Kadison and Ringrose (1997) associated with the inclusion mapping of $\mathfrak{A}$ on $\mathcal{H}$. To prove Prop. 2, we will need the following lemma characterizing $P$.

Lemma 1. $P$ is the projection onto the span of all subspaces of $\mathcal{H}_{U}$ carrying a subrepresentation of $\pi_{U}$ quasi-equivalent to $\pi$.

Proof. Let $\left(\pi_{1}, \mathcal{H}_{1}\right)$ and $\left(\pi_{2}, \mathcal{H}_{2}\right)$ be representations of $\mathfrak{A}$. Let $P_{1}, P_{2} \in$ $\overline{\pi_{U}(\mathfrak{A})}$ be their associated central projections. First, we show that if $\pi_{1}, \pi_{2}$ are quasi-equivalent, then $P_{1}=P_{2}$. The representation $\varphi_{1}$ : $A \mapsto \pi_{U}(A) P_{1}$ is quasi-equivalent to $\pi_{1}$ and $\varphi_{2}: A \mapsto \pi_{U}(A) P_{2}$ is quasi-equivalent to $\pi_{2}$. So if $\pi_{1}, \pi_{2}$ are quasi-equivalent, then so are $\varphi_{1}$ and $\varphi_{2}$. By Thm 10.3.3 (ii) of (Kadison and Ringrose, 1997, p. 736), the central carriers of $P_{1}$ and $P_{2}$ are equal, i.e. $C_{P_{1}}=C_{P_{2}}$. Since $P_{1}, P_{2}$ are central projections, $P_{1}=C_{P_{1}}=C_{P_{2}}=P_{2}$.

Next, we show that if $\pi_{1}, \pi_{2}$ are disjoint, then $P_{1} P_{2}=0$. If $\pi_{1}, \pi_{2}$ are disjoint, then so are $\varphi_{1}$ and $\varphi_{2}$-for suppose there were a subrepresentation of $\varphi_{1}$ quasi-equivalent to a subrepresentation of $\varphi_{2}$. Then we would have a subrepresentation of $\pi_{1}$ quasi-equivalent to a subrepresentation of $\pi_{2}$ (by composition of the relevant $*$-isomorphisms, see Kadison \& Ringrose 10.3.4, p. 737). Hence, by Kadison \& Ringrose 10.3.3, $P_{1} P_{2}=C_{P_{1}} C_{P_{2}}=0$.

Proposition 2. Let $V_{Q}$ be the vector subspace of $\mathfrak{A}^{* * *}$ generated by

$$
V_{0}^{Q}=\left\{\omega \in \mathfrak{A}^{*}: \omega \text { is ultraweakly continuous on } \mathcal{H}\right\}
$$

Then the $C^{*}$-algebra $\mathfrak{A}^{* *} / \sim_{V_{Q}}$ in Thm. 1 is ${ }^{*}$-isomorphic to $\mathcal{B}(\mathcal{H})$.

Proof. First, notice that by Prop. 10.1.14 of (Kadison and Ringrose, 1997, p. 722), $V_{0}^{Q}$ is the collection of functionals $\omega \in \mathfrak{A}^{*}$ such that $\omega=P \omega$, where $P \omega$ is defined as in (Kadison and Ringrose, 1997, p. $721-2)$. Let $\overline{\pi(\mathfrak{A})}$ denote the ultraweak closure of $\pi(\mathfrak{A})$.

Define $j:\left(\mathfrak{A}^{* *} / \sim_{V_{Q}}\right) \rightarrow \overline{\pi(\mathfrak{A})}$ by

$$
j([A])=\tilde{\pi}_{U}(A) P
$$

for any $A \in \mathfrak{A}^{* *}$, where $\tilde{\pi}_{U}$ is the unique weakly continuous extension of $\pi_{U}$ to $\mathfrak{A}^{* *}$. This map $j$ is well-defined because for any $A, B \in \mathfrak{A}^{* *}$, $A \sim_{V_{Q}} B$ implies $A P=B P$. 
$j$ is onto: for every $\hat{A} \in \overline{\pi(\mathfrak{A})}, \hat{A}=j([A])$ for $A=\tilde{\pi}_{U}^{-1}(\hat{A})$.

Furthermore, $j$ is one-to-one: if $A P=B P$ for $A, B \in \mathfrak{A}^{* *}$, then $[A]=[B]$.

Since $j$ obviously preserves algebraic operations, it follows that $j$ is a ${ }^{*}$-isomorphism, and since $\pi$ is quasi-equivalent to the inclusion mapping of $\mathfrak{A}$ on $\mathcal{H}$ by Thm. 10.1.12 of Kadison and Ringrose (1997), it follows that $\mathfrak{A}^{* *} / \sim_{V_{Q}}$ is ${ }^{*}$-isomorphic to $\mathcal{B}(\mathcal{H})$.

\section{References}

Arageorgis, A. (1995). Fields, particles, and curvature: Foundations and philosophical aspects of quantum field theory in curved spacetime. Ph. D. thesis, University of Pittsburgh.

Beaume, R., J. Manuceau, A. Pellet, and M. Sirugue (1974). Translation invariant states in quantum mechanics. Communications in Mathematical Physics 38, 29-45.

Bratteli, O. and D. Robinson (1996). Operator Algebras and Quantum Statistical Mechanics, Volume 2. New York: Springer.

Clifton, R. and H. Halvorson (2001). Are rindler quanta real?: Inequivalent particle concepts in quantum field theory. British Journal for the Philosophy of Science 52, 417-470.

Emch, G. (1972). Algebraic Methods in Statistical Mechanics and Quantum Field Theory. New York: Wiley.

Feintzeig, B. (2016a). On theory construction in physics: Continuity from classical to quantum. Erkenntnis, forthcoming.

Feintzeig, B. (2016b). Toward an understanding of parochial observables. British Journal for the Philosophy of science, forthcoming.

Haag, R. (1992). Local Quantum Physics. Berlin: Springer.

Halvorson, H. (2001). On the nature of continuous physical quantities in classical and quantum mechanics. Journal of Philosophical Logic 37, 27-50.

Halvorson, H. (2004). Complementarity of representations in quantum mechanics. Studies in the History and Philosophy of Modern Physics 35, 45-56. 
Halvorson, H. (2006). Algebraic quantum field theory. In J. Butterfield and J. Earman (Eds.), Handbook of the Philosophy of Physics, pp. 731-864. New York: North Holland.

Kadison, R. and J. Ringrose (1997). Fundamentals of the Theory of Operator Algebras. Providence, RI: American Mathematical Society.

Landsman, N. P. (1990). C*-algebraic quantization and the origin of topological quantum effects. Letters in Mathematical Physics 20, $11-18$.

Landsman, N. P. (1998). Mathematical Topics Between Classical and Quantum Mechanics. New York: Springer.

Landsman, N. P. (2006). Between classical and quantum. In J. Butterfield and J. Earman (Eds.), Handbook of the Philosophy of Physics, pp. 417-553. New York: North Holland.

Petz, D. (1990). An Invitation to the Algebra of Canonical Commutation Relations. Leuven: Leuven University Press.

Ruetsche, L. (2002). Interpreting quantum field theory. Philosophy of Science 69(2), 348-378.

Ruetsche, L. (2003). A matter of degree: Putting unitary inequivalence to work. Philosophy of Science 70(5), 1329-1342.

Ruetsche, L. (2006). Johnny's so long at the ferromagnet. Philosophy of Science 73(5), 473-486.

Ruetsche, L. (2011). Interpreting Quantum Theories. New York: Oxford University Press.

Sakai, S. (1971). $C^{*}$-algebras and $W^{*}$-algebras. New York: Springer.

Summers, S. (1999). On the stone-von neumann uniqueness theorem and its ramifications. In M. Redei and M. Stoeltzner (Eds.), John von-Neumann and the Foundations of Quantum Physics, pp. 135152. Dordrecht: Kluwer.

Wald, R. (1994). Quantum Field Theory in Curved Spacetime and Black Hole Thermodynamics. Chicago: University of Chicago Press. 